\title{
Parathyroid hormone induces formation of new cancellous bone with substantial mechanical strength at a site where it had disappeared in old rats
}

\author{
Hans Oxlund, Michel Dalstra ${ }^{1}$, Charlotte Ejersted and Troels T Andreassen \\ Department of Connective Tissue Biology, Institute of Anatomy and ${ }^{1}$ Orthopaedic Research Laboratory, University of Aarhus, DK-8000 Aarhus C, \\ Denmark \\ (Correspondence should be addressed to H Oxlund; Email: ho@ana.au.dk)
}

\begin{abstract}
Objective: The present study addresses the question - can PTH induce formation of trabeculae in areas where cancellous bone has disappeared? Two-year-old male rats were chosen, because in this aged animal model the distal femurs have almost no cancellous bone, and the marrow cavity has reached a substantial dimension.

Design: The rats were injected for 56 days with either PTH(1-34), $15 \mathrm{nmol} / \mathrm{kg} /$ day $(62.5 \mu \mathrm{g} / \mathrm{kg} /$ day $)$, or vehicle.

Methods: Transverse specimens, 2-mm high, were cut from the distal femoral metaphysis. Marrow cavity diameters and cancellous bone trabeculae were analysed by a micro-computerized tomography scanner. The cancellous bone within the cortical and endocortical rim of each specimen was submitted to a biomechanical compression test. Furthermore, the cancellous bone was studied by dynamic tetracycline labelling and histomorphometry.

Results: In the vehicle-injected group the trabecular bone volume was 0\% (0-1.4), median (range). All PTH-injected rats had trabeculae in the distal metaphysis and the trabecular bone volume $(6.7 \%$ $(2.3-12.0))$ was markedly increased $(P<0.003)$. The median trabecular thickness was increased $(P<0.003)$ in the PTH-injected rats $(118 \mu \mathrm{m}(104-125))$ compared with the vehicle group $(0 \mu \mathrm{m}(0-71))$. The compressive stress was increased $(P<0.003)$ in the PTH-injected group (0.7 MPa (0.1-2.1)) compared with the vehicle-injected group (0 MPa (0-0.4)). The histomorphometry revealed that only 3 animals of the 10 in the vehicle-injected group had trabeculae in the distal femoral metaphysis. All PTH-injected animals (12 of 12) had continuous trabecular bone network in the marrow cavity.

Conclusion: Intermittent PTH treatment induced marked formation of new cancellous bone trabeculae with substantial mechanical strength, at a site where it had disappeared in old rats.
\end{abstract}

European Journal of Endocrinology 146 431-438

\section{Introduction}

Rat models are increasingly used in screening studies of agents for prevention and treatment of osteoporosis. Besides determination of bone mass and bone formation rate, it has been recommended to perform biomechanical tests to ensure proper biomechanical competence of the bone tissue (1-3). In human osteoporotic individuals the bone loss is especially pronounced in the cancellous bone of the spine and of the femoral neck. Trabeculae become thinner and some trabeculae are resorbed and disappear during the life-long remodelling processes. It is the general view that lost trabeculae cannot regenerate and that cancellous bone structures cannot be restored in a skeletal site where it has disappeared, for example in the vertebral bodies and femoral neck of osteoporotic patients. In screening studies using rat models, parathyroid hormone (PTH) injected intermittently has shown remarkable ability to stimulate new bone formation on endocortical and cancellous bone surfaces (4-8). The increased bone formation has been delineated by determination of bone mass, bone formation rate by dynamic histomorphometry, and biomechanical testing of bone specimens prepared from vertebral bodies, femoral neck and femoral middiaphyses. In these studies the new bone formation takes place on existing surfaces. Furthermore, the biomechanical tests have all been performed on cortical bone or combinations of cortical and cancellous bone. The rat femoral mid-diaphysis is totally comprised of cortical bone and the rat femoral neck is predominantly 
composed of cortical bone. Even if there is a substantial amount of cancellous bone in the rat vertebral body, the relatively thick cortical shell bears a substantial part $(72 \%)$ of the load in compression tests of the rat vertebral body (9). The role of the cortical shell in load-bearing during the compression test of vertebral body specimens is even more predominant in old and in ovariectomised rats, where there has been loss of cancellous bone. Hogan et al. (10) have published a direct compression test of pure cancellous bone in the proximal rat tibia. The cancellous bone was compressed between two platens of the same size. The cancellous bone is not, however, homogeneous; the structure is looser in the axial centre and a denser structure is found peripherally. Therefore, it is necessary to use platens, which compress both the central and the peripheral parts of the cancellous bone. This means sets of platens of increasing size, so that a correct set of platens can be chosen corresponding to the actual diameter of the cancellous bone in each specimen. Using only one set of platens results in a systematic error, because only the central structurally looser part will be compressed in large specimens, while the peripheral denser part will also be included in the compression test of small specimens. The problem is accentuated in the ovariectomised rat model, where it is well known that ovariectomy results in growth and modelling of bones, besides the loss of cancellous bone (11). This inhomogeneity also influences the indentation tests (12-14), where an indenter, $1.6 \mathrm{~mm}$ thick (fixed size), compresses and penetrates the cancellous bone.

The present study has addressed the question - can PTH induce formation of new bone trabeculae at a site where the cancellous bone has disappeared? Two-yearold male rats were chosen because they have distal femurs with relatively large marrow cavities, where the cancellous bone by that age has disappeared in most animals. After PTH injections, $15 \mathrm{nmol} / \mathrm{kg} /$ day $(62.5 \mu \mathrm{g} / \mathrm{kg} /$ day $)$ for 56 days, the marrow cavity diameters and cancellous bone structure in specimens of the distal femoral metaphysis were analysed by a micro-computerized tomography (CT) scanner. Using these data, a set of platens corresponding to the diameters of the marrow cavity was chosen and used in the compression test of the cancellous bone. Furthermore, dynamic histomorphometry and histological studies of the marrow cavity and cancellous bone were performed in the distal femoral metaphysis of old vehicle- and PTH-injected rats.

\section{Materials and methods}

Twenty-one 24-month-old male Sprague-Dawley rats (IFFA Credo l'Arbresle, Cedex, France) were divided at random into two groups. The rats were fed standard pellets (Altromin, Chr. Pedersen, Ringsted, Denmark), containing $0.9 \%$ calcium, $0.7 \%$ phosphorous and 1 I.E. vitamin $\mathrm{D}_{3}$. Synthetic human parathyroid hormone (PTH(1-34)) was obtained from Bachem Inc (Torrance, CA, USA) and prepared in a vehicle of $0.15 \mathrm{~mol} / \mathrm{l}$ saline with $2 \%$ rat serum albumin (Sigma, St. Louis, MO, USA) and $0.001 \mathrm{~mol} / \mathrm{l} \mathrm{HCl}$. The rats were injected daily subcutaneously for 56 days with either $15 \mathrm{nmol} / \mathrm{kg}$ $(62.5 \mu \mathrm{g} / \mathrm{kg}) \operatorname{PTH}(1-34)(n=11)$ or vehicle $(n=10)$. The rats injected with vehicle constituted the control group. Six 9-month-old (adult) rats with known intact cancellous bone in the distal femur were included. The rats were anaesthetized by pentobarbital (SAD, Copenhagen, Denmark) and then killed by exsanguination. The hind legs were exarticulated at the hip joints and stored airtight at $-20^{\circ} \mathrm{C}$.

\section{Preparation of specimens from the distal femoral metaphysis for determination of cancellous bone volume and cancellous bone compression strength of the specimens}

The femurs were dissected free and the length of each femur measured from the caput femoris to the condylus

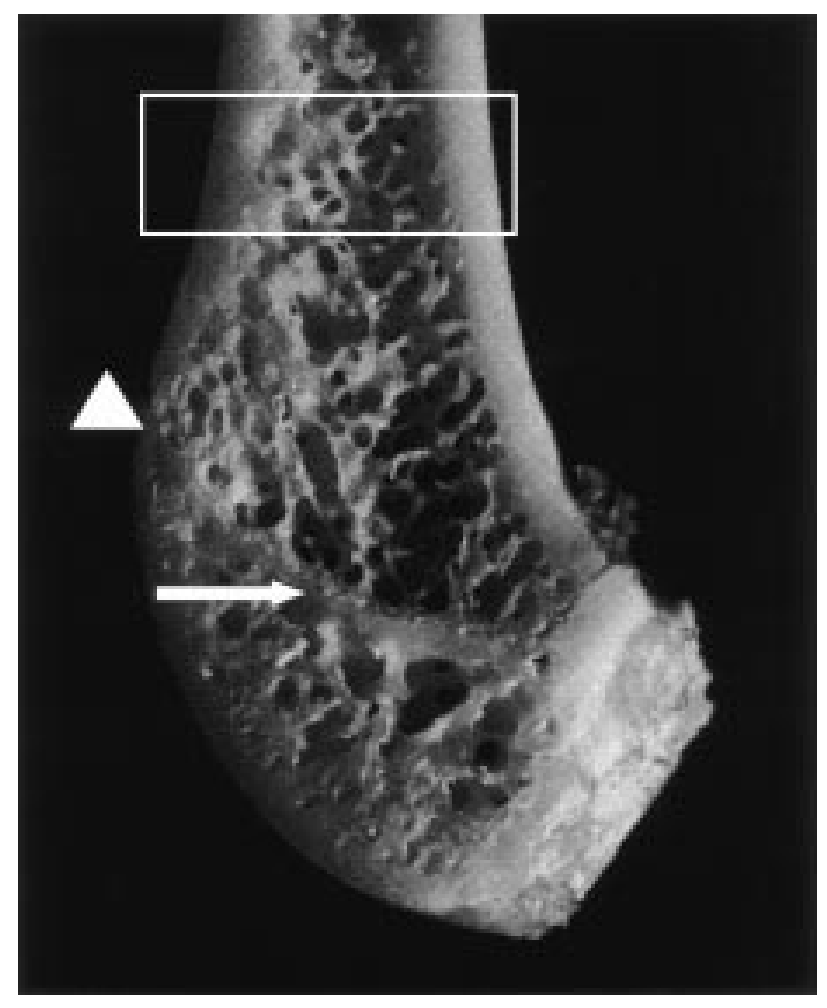

Figure 1 Micro photo of a sagittal section prepared from the distal femur of a 3-month-old rat, illustrating cortical bone and abundant cancellous bone with continuous trabeculae in the distal part of the metaphysis. The distal epiphyseal growth plate is marked by a white arrow, the proximal edge of the facies patellaris femoris is marked by a white triangle and a two-mm-high transverse specimen for the micro-CT-scanner analysis and biomechanical compression test of the cancellous bone inside the cortical rim is marked by a rectangular box. 
medialis. A 2.0-mm-high specimen (box in Fig. 1) from the distal femoral metaphysis was cut transversely $3.0 \mathrm{~mm}$ proximally to the edge (marked by a triangle in Fig. 1) of facies articularis patellaris. The distal femoral epiphyseal growth plate is marked by an arrow. Care was taken to obtain plano-parallel proximal and distal surfaces of each specimen by using a precision bone saw (Exakt-Apparatebau, Otto Herrmann, Norderstedt, Germany). The transverse diameter of the cancellous bone minus endocortical bone was determined for each specimen using a micro-CTscanner ( $\mu$-CT20, Scanco Medical, Bassersdorf, Switzerland). The samples were placed horizontally in a sample holder and kept moist during scanning. The actual in-plane resolution of the images was $30 \mu \mathrm{m}$ and a total of 85 scans was required to cover each sample. Besides the diameter of the marrow cavity minus endocortical bone, the trabecular bone volume of the cancellous bone cylinder and the mean trabecular thickness were determined using the micro-CTscanner's built-in software.

\section{Cancellous bone compression analysis}

The cancellous bone of the 2.0-mm-thick specimens was placed between two platens in a materials testing machine (Alwetron 250, Lorentzen \& Wettre, Stockholm, Sweden). The set-up for cancellous bone compression analysis, as used in the present study, is shown schematically in Fig. 2. The method is a modification of the method used by Hogan et al. (10) on cancellous bone in the proximal tibia. The set of platens used corresponded to the transverse diameter of the marrow cavity including cancellous bone minus endocortical bone, determined as above by the micro-CT-scanner for each specimen. Ten sets of platens were used with diameters ranging from 1.2 to $2.2 \mathrm{~mm}$ and an increment of $0.1 \mathrm{~mm}$. Each $2.0-\mathrm{mm}$-thick specimen was placed in the materials testing machine between the two platens. The upper platen was lowered at a constant speed of $1.0 \mathrm{~mm} / \mathrm{min}$, compressing the cancellous bone between the upper and lower platen. Load and deformation values were recorded continuously by transducers coupled to measuring bridges, and the signals were fed to an $x-y$ recorder. The cancellous bone was compressed until fracture of the cancellous bone structure. The load-deformation curves obtained were scanned into a computer and the following parameters were calculated using the SigmaScan software (Jandel Scientific Software, St. Rafael, CA, USA) (Fig. 3): ultimate compressive load (Ucl), ultimate stiffness (slope of the linear part of the load-deformation curve, i.e. tangent to the angle A), deformation at fracture (Df), energy absorption at fracture (area between the curve and the deformation-axis).

\section{CANCELLOUS BONE COMPRESSIVE STRENGTH}

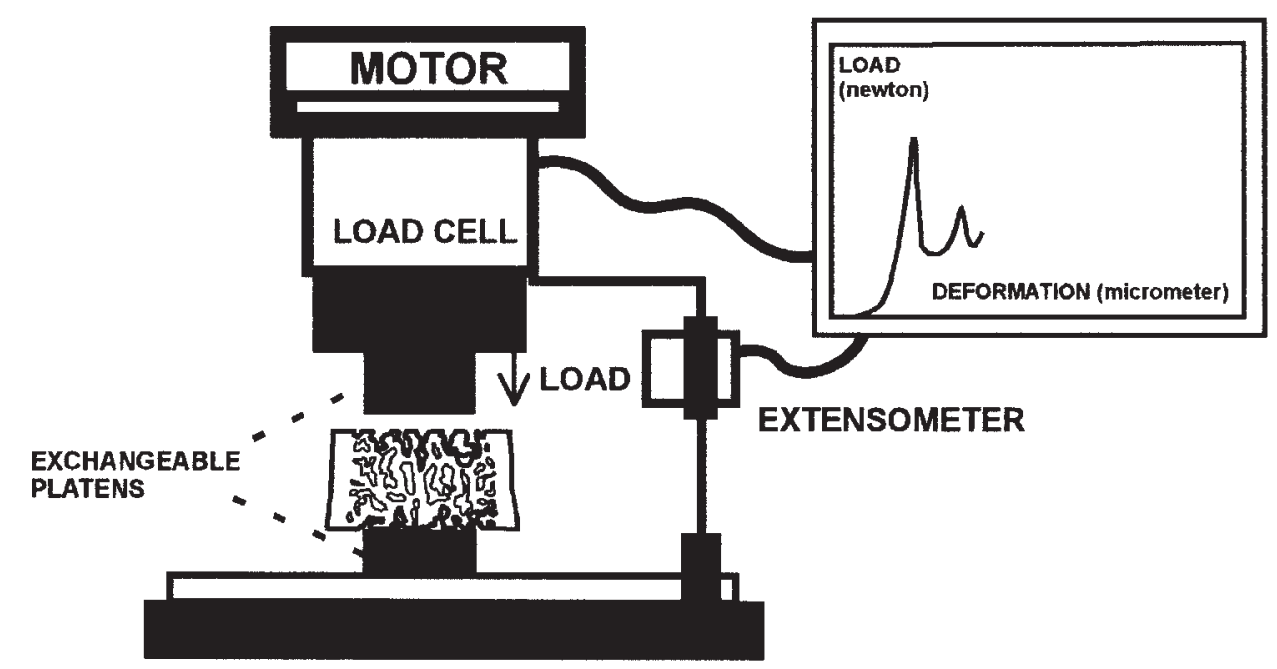

Figure 2 Schematic drawing of the cancellous bone compression test. Each 2.0-mm-high specimen was placed in the materials testing machine between two platens of equal size corresponding to the cancellous bone. The upper platen was lowered at a constant speed of $1.0 \mathrm{~mm}$ per min, compressing the cancellous bone between the upper and lower platens. Load and deformation values were recorded continuously by transducers and the signals recorded as a load-deformation curve. 


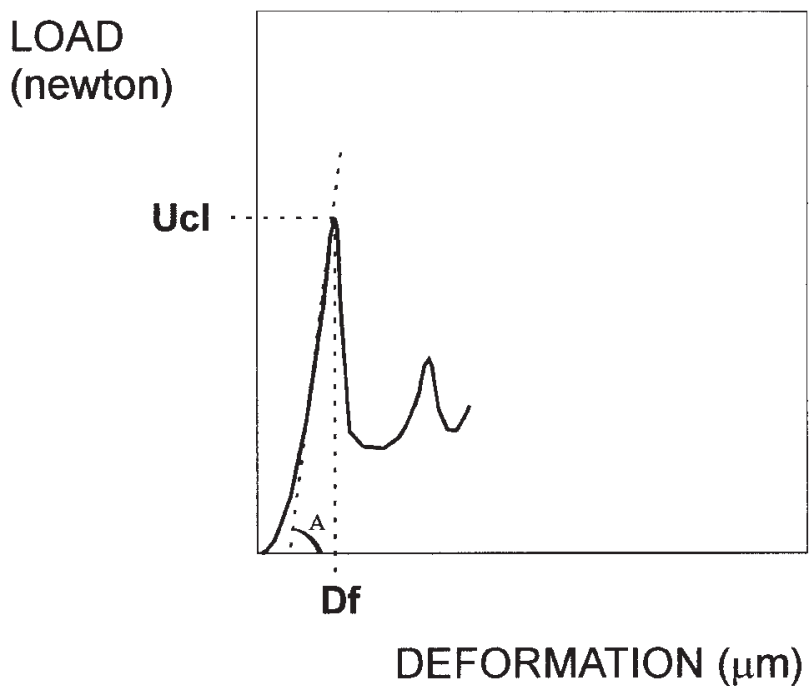

Figure 3 Transverse specimens of the distal femoral metaphysis were prepared and the cancellous bone within the rim of cortical bone was compressed in a materials testing machine. A typical load-deformation curve resulting from such a test is shown in the diagram. Ucl is ultimate compressive load, the slope of the linear part of the load-deformation curve (tangent to the angle $A$ ) is the stiffness of the cancellous bone. Df on the deformation axis is deformation in micrometers at fracture, and the area between the curve and the deformation axis is the energy required to fracture the cancellous bone between the two platens.

\section{Cancellous bone mechanical properties}

Ultimate compressive stress and ultimate stiffness (Young's modulus) were calculated by normalising the ultimate compressive load and ultimate stiffness values to the cross-sectional area of the cancellous bone compressed $(15,16)$, i.e. the platen area of each specimen, giving ultimate compressive stress $(\mathrm{MPa})$, Young's modulus $(\mathrm{MPa})$ and energy absorption (MPa $\times$ $\mu \mathrm{m})$.

\section{Adapted platen size versus fixed platen size}

Fourteen one-year-old female rats were divided randomly into two groups: a group for compression of the cancellous bone in the distal femoral metaphysis using fixed platen size $(n=7)$ and a group for compression of the cancellous bone using platen sizes corresponding to the diameter of the cancellous bone $(n=7)$. Preparation of the bone samples was performed as described. The size of the fixed platens was chosen corresponding to the sample possessing the smallest diameter. The fixed size platen test resulted in a compressive stress of $0.23 \pm 0.08 \mathrm{MPa}$ (mean \pm S.E.M.). The adapted size platen test resulted in a mean stress value of $0.39 \pm 0.09 \mathrm{MPa}$. Consequently, higher stress values and a lower coefficient of variation are obtained when applying a platen size which actually corresponds to the diameter of the cancellous bone in each sample. Therefore, the procedure of the adapted platen size was used in the present experiments.

\section{Dynamic histomorphometry of the distal femoral metaphysis}

Male Wistar rats, 21 months old, were randomly divided into two groups, a vehicle-injected $(n=10)$ and a PTH-injected $(n=12)$ group. The PTH preparation, dose, vehicle and standard rat pellet chow diet were as described above. The PTH injections were given daily for 56 days. On day 35 and day 49 of the treatment, the rats were given intraperitoneal injections of $15 \mathrm{mg} / \mathrm{kg}$ tetracycline (Sigma) and $15 \mathrm{mg} / \mathrm{kg}$ calcein (Sigma) respectively, to label mineralization surfaces. The rats were killed by exsanguination under pentobarbital anaesthesia. Two-mm-high transverse samples were cut from the distal femoral metaphysis as described above. The samples were fixed in $70 \%$ ethanol at $4{ }^{\circ} \mathrm{C}$ for undecalcified bone processing. The samples were dehydrated with sequential changes of ethanol and embedded in methyl methacrylate. Sagittal sections, $150 \mu \mathrm{m}$ thick, were cut by means of a precision bone saw with a $100-\mu \mathrm{m}$-thick diamond blade, and 8- $\mu \mathrm{m}$-thick sections were cut using a microtome (Jung RM2065, Leica Instruments $\mathrm{GmbH}$, Nussloch, Germany). Some of the sections were then studied in the epifluorescence microscope (Leica DMRB, Leica Mikroskopie \& Systeme $\mathrm{GmbH}$, Wetzlar, Germany) at a magnification of $\times 50$ to $\times 400$. Other sections were stained with toluidine blue or Masson-Goldener-Trichrome (17) and the cancellous bone was studied at a magnification of $\times 100$ to $\times 400$.

\section{Statistical analysis}

Data of body weight and femur lengths were normally distributed and presented as group mean values with S.E.M. Differences were evaluated by Student's $t$-test. Data of cancellous bone analyses were not normally distributed and were therefore presented as median values with range in parentheses. The Mann-Whitney's U-test was used for testing differences between the two groups. The presence of cancellous bone in the distal femoral metaphysis of the PTH-injected versus the vehicle-injected animals, was evaluated by the Fisher exact test. $P<0.05$ (two-tailed) was considered statistically significant (18).

\section{Results}

The body weights and the lengths of the femurs were not influenced by the PTH treatment. The mean body weight of the vehicle-injected group was $675 \pm 19 \mathrm{~g}$ (mean value \pm S.E.M.) at the start of the injection 
period, and $643 \pm 30 \mathrm{~g}$ at the end. In the PTH group, the mean body weight was $680 \pm 19 \mathrm{~g}$ at the start of the experiment and $661 \pm 21 \mathrm{~g}$ at the end. The length of the femur was $41.7 \pm 0.3 \mathrm{~mm}$ in the vehicle-injected group and $41.8 \pm 0.2 \mathrm{~mm}$ in the PTH-injected group. The distal femoral metaphysis samples were slightly

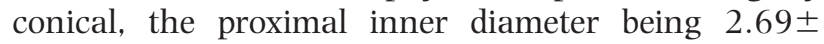
$0.09 \mathrm{~mm}$, mean \pm S.E.M., and distal diameter $2.91 \pm$ $0.09 \mathrm{~mm}$ in the vehicle group. In the PTH-injected group, the proximal inner diameter was $2.48 \pm$ $0.05 \mathrm{~mm}$ and the distal diameter $2.73 \pm 0.06 \mathrm{~mm}$, i.e. $10 \%$ larger $(P<0.05)$ than the proximal diameter.

Only 3 of 10 vehicle-injected rats had detectable trabeculae in their distal metaphyses and a trabecular bone volume of $0 \%(0-1.4)$, median (range). All PTH-injected rats had trabeculae in their distal metaphyses and the trabecular bone volume $(6.7 \%$ $(2.3-12.0))$ was markedly increased $(P<0.003)$. Consequently, the number of PTH-injected rats with cancellous bone in the distal metaphysis differed significantly $(P=0.002$, Fisher exact test $)$ from the vehicle-injected rats. Likewise, the cancellous bone compressive stress, Young's modulus and energy absorption were zero or very low in the vehicle-injected group and markedly increased in the PTH-injected group (Table 1). The median trabecular thickness was increased in the PTH-injected rats $(118 \mu \mathrm{m}$ (104-125)) compared with the vehicle-injected group $(0 \mu \mathrm{m}(0-71))$.

The histomorphometry showed absence of cancellous bone (Fig. 4) in most samples from the vehicle-injected animals (7 of 10) or dispersed fragments of the cancellous bone without continuity ( 2 of 10 ). Only one vehicle-injected animal had cancellous bone in the marrow cavity as a thin continuous network. In the samples from animals injected with PTH, cancellous bone structures were found with trabeculae in a continuous network through the marrow cavity (12 of $12)$, a significant difference $(P<0.001)$. The PTH injections resulted in the formation of a new cancellous bone network. The epifluorescence microscopy of tetracycline and calcein labellings at day 35 and day 49 of the treatment period, and distinct difference in colour between the tetracycline and calcein labellings, enabled evaluation of the formation of trabeculae in relation to the day of labelling. By far the majority of the trabeculae had been formed after day 35 of the treatment period (Fig. 4).

\section{Discussion}

The rat distal femoral metaphysis is a bone site with a relatively large amount of cancellous bone in young and adult rats. A pronounced loss of cancellous bone is seen in relation to aging as illustrated in Fig. 4. Therefore, the distal femoral metaphysis is an appropriate site for studies of cancellous bone regeneration in the aged rat model. PTH was shown to induce formation of new cancellous bone trabeculae in this site.

Osteoblasts, bone lining cells and bone marrow stroma cells which can differentiate into the osteoblast cell lineage have PTH receptors. PTH stimulates these cells through modulation of cAMP concentrations and cAMP-dependent protein kinases (19). The rapid increase in bone formation induced by intermittent PTH does not require cell proliferation and seems to be the result of activation of pre-existing bone lining cells to osteoblasts $(20,21)$. The anabolic effects of PTH on bone formation is local; insulin-like growth factor I (IGF-I) and transforming growth factor (TGF)beta- 1 are local mediators $(22-25)$. PTH seems to stimulate bone formation regardless of bone marrow composition; thus PTH may increase the bone formation rate markedly in the distal tibial metaphysis although it contains yellow bone marrow (26). PTH has also been shown to stimulate production of prostaglandins from rat osteogenic cells resulting in stimulated bone formation (27). Furthermore, PTH may stimulate bone marrow osteoprogenitor cells to proliferate, migrate and differentiate into osteoblasts if the bone tissue is loaded normally $(28,29)$. This process may be the one that enables formation of new cancellous bone in the distal femoral diaphysis of the old rats in the present study.

Most studies intending to test the biomechanical strength of cancellous bone in rat models actually test bone specimens containing both cortical and cancellous bone $(4,15,30-32)$. Even if the proportion of

Table 1 Biomechanical competence of cancellous bone in the distal femoral metaphysis of old rats injected with PTH. Results are median values with range in parentheses.

\begin{tabular}{lcccc}
\hline Group & $\boldsymbol{n}$ & Compressive stress $(\mathrm{MPa})$ & Young's modulus $(\mathrm{MPa})$ & Energy at fracture $(\mathrm{Mpa} \times \mu \mathrm{m})$ \\
\hline Adult rats & 6 & 1.4 & 34 & 42 \\
9 months old & & $(0.4-4.0)$ & $(3-123)$ & $(13-126)$ \\
Vehicle control & 10 & 0 & 0 & 0 \\
27 months old & & $(0-0.4)$ & $(0-1)$ & $(0-27)$ \\
PTH-injected & 11 & $0.7^{*}$ & $18^{*}$ & $38^{*}$ \\
27 months old & & $(0.1-2.1)$ & $(1-58)$ & $(16-101)$ \\
\hline
\end{tabular}

${ }^{*} P<0.003$ compared with vehicle control. 


\section{Vehicle}
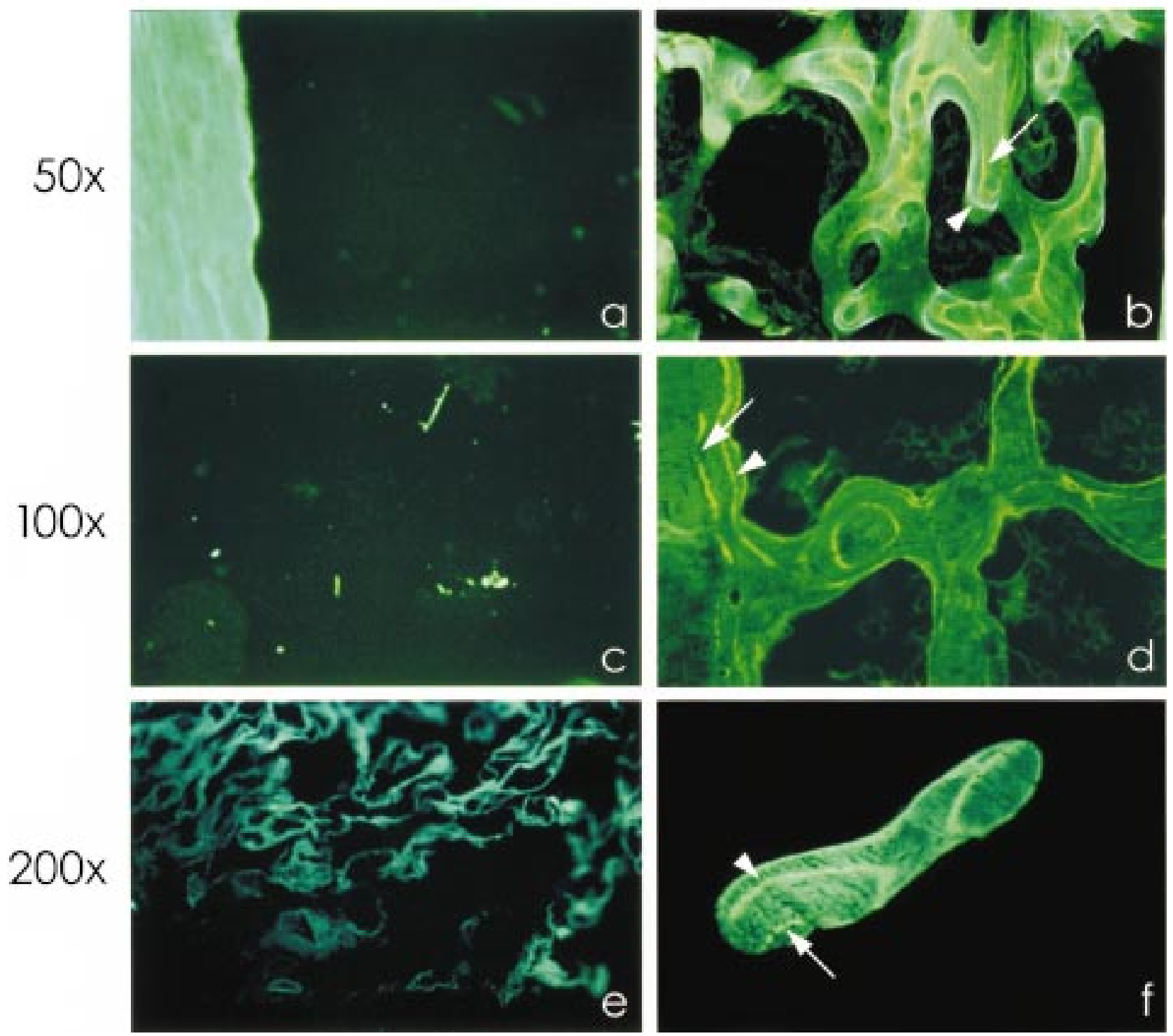

Figure 4 Micro photos of cancellous bone prepared from undecalcified distal femoral metaphyses of vehicle-injected 27-month-old rats (left column) and PTH-injected 27-month-old rats (right column). Tetracycline was injected on day 35 (yellow line, arrow) and calcein on day 49 (white line, arrowhead) of the 56-day treatment with PTH. The 27-month-old rats injected with vehicle had no cancellous bone in the marrow cavity $(a, c, e)$. Cortical bone is seen to the left in (a). Bone marrow stroma is seen in (e). The PTH-injected rats had cancellous bone consisting of well-connected trabeculae (b, d, f). Cortical bone is seen to the left in (d).

the cancellous bone in rat vertebral bodies is relatively high, there is still a considerable cortical rim or shell that carries a large percentage of the load in compression tests, and in vertebral bodies from old or ovariectomised rats most of the load is carried by the cortical shell. Therefore, the compression test of vertebral body specimens cut transversely cannot be claimed to be a test of cancellous bone only. The proportion of cancellous bone in the rat femoral neck specimens is even lower because of the relatively thick cortical wall in the rat femoral neck; consequently the test of rat femoral neck as reported by Søgaard et al. (30) and Peng et al. (31) is not a test of cancellous bone biomechanical properties, but a test of the whole femoral neck structure of cortical and cancellous bone. In young and adult rats there are relatively large amounts of cancellous bone in the distal femoral metaphysis. Specimens cut transversely from that region, $4 \mathrm{~mm}$ high, were tested in compression (32). This approach is also a test of both cortical and cancellous bone, 
and studies in our laboratory have shown that most of the load is carried by the cortical shell (9). This is even more pronounced in old rats where the cancellous bone disappears in the distal femoral metaphysis. In tests of the distal femoral metaphysis another test method has been used that certainly tests only cancellous bone. That is the indentation test, where an indentor, $1.6 \mathrm{~mm}$ thick, compresses and penetrates to a depth of $2.0 \mathrm{~mm}$ the cancellous bone of a 4.0 -mm-high transverse specimen placed on a flat surface $(13,14)$. The cancellous bone of the distal metaphysis is not, however, uniform. The density of trabeculae is less in the central compared with the peripheral part of the cancellous bone. Therefore, using an indentor with a fixed thickness $(1.6 \mathrm{~mm})$ results in a systematic error when studying large and small rats, because in small rats a larger part of the more dense peripheral part of the cancellous bone will be compressed compared with larger rats where possibly only the central part with less trabecular density will be compressed. This causes a problem whenever the size of one group of rats differs compared with another group. Furthermore, compression and indentation on the upper surface of the cancellous bone of the specimen, which is fully supported by a flat surface on the lower side of the specimen, is a biomechanically complicated set up, where it is very difficult to know which trabeculae are compressed, and difficult to calculate material properties. Furthermore, the forces created during the penetration are very complicated or incalculable.

Only cancellous bone is compressed in the test method of the present paper. A micro-CT scanner was used to analyse the three-dimensional structure of each specimen prepared from the distal femoral metaphysis. The transverse diameter of the cancellous bone was determined and used for selection of the set of platens, which were mounted in the materials testing machine (Fig. 2). Then the specimen was carefully placed between the two platens and compressed. The load and deformation were continuously registered as a load-deformation curve for each specimen (Fig. 3). The cancellous bone withstood deformations of $57-150 \mu \mathrm{m}$ until the cancellous bone structure fractured and collapsed at the point of maximum load. The upper and lower platens were of the same size. Therefore, we knew the cross-sectional area of the compressed cancellous bone, and could calculate stress values, Young's modulus and energy absorption for each specimen. The cancellous bone in compression stays in situ, and the trabeculae keep their lateral connections intact. This may influence the compression test by counteracting lateral expansion of the cancellous bone during the compression. On the other hand, it may be argued that the cancellous bone stays in situ and is supported laterally as it is in vivo. Using the present method, it is an absolute condition that a correct measure of the cancellous bone diameter is obtained and that the size of platens are chosen accordingly, in order to avoid incorporation of endocortical bone in the compression test.

\section{Acknowledgements}

We acknowledge funding from The Danish Health Research Council, grants no. 9500922 and 9600822 (Aarhus University-Novo Nordisk Centre for Research in Growth and Regeneration) and 52-00-1034, Aarhus University Research Foundation, Novo Nordisk Foundation. The skilled technical assistance of A Berg, $\mathrm{C}$ Knæhus and E K Mikkelsen, and linguistic revision of M Fischer are gratefully acknowledged.

\section{References}

1 Thompson DD, Simmons HA, Pirie CM \& Ke HZ. FDA guidelines and animal models for osteoporosis. Bone $199517125 \mathrm{~S}-133 \mathrm{~S}$.

2 Kimmel DB. Animal models for in vivo experimentation in osteoporosis research. In Osteoporosis, pp 671-690. Eds R Marcus, D Feldman \& J Kelsey. San Diego, CA: Academic Press, 1996.

3 Mosekilde L \& Reeve J. Treatment with PTH peptides. In Osteoporosis, pp 1293-1311. Eds R Marcus, D Feldman \& J Kelsey. San Diego, CA: Academic Press, 1996.

4 Mosekilde LI, Søgaard CH, Danielsen CC, Tørring O \& Nilsson MHL. The anabolic effects of human parathyroid hormone (hPTH) on rat vertebral body mass are also reflected in the quality of bone, assessed by biomechanical testing: a comparison study between hPTH-(1-34) and hPTH-(1-84). Endocrinology 1991 $129421-428$.

5 Dempster DW, Cosman F, Parisien M, Shen V \& Lindsay R. Anabolic actions of parathyroid hormone on bone. Endocrine Review 199314 690-709.

6 Ejersted C, Andreassen TT, Oxlund H, Jørgensen PH, Bak B, Häggblad J et al. Human parathyroid hormone (1-34) and (1-84) increase the mechanical strength and thickness of cortical bone in rats. Journal of Bone and Mineral Research $1993 \mathbf{8}$ 1097-1101.

7 Ejersted C, Andreassen TT, Nilsson MHL \& Oxlund H. Human parathyroid hormone(1-34) increases bone formation and strength of cortical bone in aged rats. European Journal of Endocrinology $1994130201-207$.

8 Oxlund H, Ejersted C, Andreassen TT, Tørring O \& Nilsson MHL. Parathyroid hormone (1-34) and (1-84) stimulate cortical bone formation both from periosteum and endosteum. Calcified Tissue International $1993 \mathbf{5 3} 394-399$.

9 Oxlund H, Ørtoft G \& Andreassen TT. Effects of growth hormone on vertebral cancellous bone compressive strength in old rats. Bone 199823 S583.

10 Hogan HA, Ruhmann SP \& Sampson HW. The mechanical properties of cancellous bone in the proximal tibia of ovariectomized rats. Journal of Bone and Mineral Research 200015 284-292.

11 Kalu DN. The ovariectomised rat model of postmenopausal bone loss. Bone and Mineral 199115 175-191.

12 Hvid I, Andersen K \& Olesen S. Cancellous bone strength measurements with the osteopenetrometer. Engineering in Medicine 198413 73-78.

13 Shen V, Birchman R, Xu R, Otter M, Wu D, Lindsay R et al. Effects of reciprocal treatment with estrogen and estrogen plus parathyroid hormone on bone structure and strength in ovariectomised rats. Journal of Clinical Investigation $1995 \mathbf{9 6}$ $2331-2338$.

14 Meng XW, Liang XG, Birchman R, Wu DD, Dempster DW, Lindsay $\mathrm{R}$ et al. Temporal expression of the anabolic action of PTH in 
cancellous bone of ovariectomised rats. Journal of Bone and Mineral Research 199611 421-429.

15 Andreassen TT, Melsen F \& Oxlund H. The influence of growth hormone on cancellous and cortical bone of the vertebral body in aged rats. Journal of Bone and Mineral Research 199611 $1094-1102$.

16 Turner $\mathrm{CH} \&$ Burr DB. Basic biomechanical measurements of bone: a tutorial. Bone 199314 595-608.

17 Baron R, Vignery A, Neff L, Silverglade A \& Santa Maria A. Processing of undecalcified bone specimens for bone histomorphometry. In Bone Histomorphometry: Techniques and Interpretation, pp 13-35. Ed. RR Recker. Boca Raton, Florida: CRC Press, Inc, 1990

18 Sokal RR \& Rohlf FJ. Biometry. San Francisco: WH Freeman, 1981

19 Boguslawski G, Hale LV, Yu XP, Miles RR, Onyia JE, Santerre RF et al. Activation of osteocalcin transcription involves interaction of protein kinase A- and protein kinase C-dependent pathways. Journal of Biological Chemistry 200014 999-1006.

20 Dobnig H \& Turner RT. Evidence that intermittent treatment with parathyroid hormone increases bone formation in adult rats by activation of bone lining cells. Endocrinology 1995136 $3632-3638$

21 Schmidt IU, Dobnig H \& Turner RT. Intermittent parathyroid hormone treatment increases osteoblast number, steady state messenger ribonucleic acid levels for osteocalcin, and bone formation in tibial metaphysis of hypophysectomized female rats. Endocrinology $19951365127-5134$.

22 Canalis E, Centrella M, Burch W \& McCarthy TL. Insulin-like growth factor I mediates selective anabolic effects of parathyroid hormone in bone cultures. Journal of Clinical Investigation 1989 $8360-65$.

23 McCarthy TL, Centrella M \& Canalis E. Parathyroid hormone enhances the transcript and polypeptide levels of insulin-like growth factor I in osteoblast-enriched cultures from fetal rat bone. Endocrinology $1989 \mathbf{1 2 4} 1247-1253$.

24 Pfeilschifter J, Laukhuf F, Muller-Beckmann B, Blum WF, Pfister T \& Ziegler R. Parathyroid hormone increases the concentration of insulin-like growth factor-I and transforming growth factor beta 1 in rat bone. Journal of Clinical Investigation 199596 $767-774$.
25 Watson P, Lazowski D, Han V, Fraher L, Steer B \& Hodsman A. Parathyroid hormone restores bone mass and enhances osteoblast insulin-like growth factor I gene expression in ovariectomised rats. Bone $199516357-365$

26 Li M, Liang H, Shen Y \& Wronski TJ. Parathyroid hormone stimulates cancellous bone formation at skeletal sites regardless of marrow composition in ovariectomised rats. Bone $1999 \mathbf{2 4}$ 95-100.

27 Mori S, Jee WS \& Li XJ. Production of new trabecular bone in osteopenic ovariectomised rats by prostaglandin $\mathrm{E}_{2}$. Calcified Tissue International $1992 \mathbf{5 0} 80-87$.

28 Nishida S, Yamaguchi A, Tanizawa T, Endo N, Mashiba T, Uchiyama $\mathrm{Y}$ et al. Increased bone formation by intermittent parathyroid hormone administration is due to the stimulation of proliferation and differentiation of osteoprogenitor cells in bone marrow. Bone $199415717-723$.

29 Kostenuik PJ, Harris J, Halloran BP, Turner RT, Morey-Holton ER \& Bikle DD. Skeletal unloading causes resistance of osteoprogenitor cells to parathyroid hormone and to insulin-like growth factor-I. Journal of Bone and Mineral Research 199914 $21-31$.

30 Søgaard CH, Wronski TJ, McOsker JE \& Mosekilde L. The positive effect of parathyroid hormone on femoral neck bone strength in ovariectomised rats is more pronounced than that of estrogen or bisphosphonates. Endocrinology 1994134 $650-657$.

31 Peng Z, Tuukkanen J. Zhang H \& Vaananen HK. Alteration in the mechanical competence and structural properties in the femoral neck and vertebrae of ovariectomised rats. Journal of Bone and Mineral Research 199914 616-623.

32 Mosekilde L, Thomsen JS, Orhii PB \& Kalu DN. Growth hormone increases vertebral and femoral bone strength in osteopenic, ovariectomised, aged rats in a dose-dependent and site-specific manner. Bone 199823 343-352.

Received 6 October 2001

Accepted 5 December 2001 